\title{
A supportive pedagogical package for distance learning and remote laboratories
}

\author{
Geneviève Samson ${ }^{1}$, Jean-Sébastien Deschênes ${ }^{1, *}$, Hamdjatou Kane ${ }^{2}$, Rhadi Mhiri ${ }^{3}$, Vahe Nerguizian ${ }^{3}$, \\ Hamadou Saliah-Hassane ${ }^{4}$ \\ ${ }^{1}$ Université du Québec à Rimouski, 300 Allée des Ursulines, Rimouski, QC, Canada \\ ${ }^{2}$ Université du Québec en Outaouais, 283 Boul. Alexandre-Taché, Gatineau, QC, Canada \\ ${ }^{3}$ École de Technologie Supérieure, 1100 rue Notre-Dame Ouest, Montréal, QC, Canada \\ ${ }^{4}$ Télé-Université du Québec, 100 rue Sherbrooke Ouest, Montréal, QC, Canada \\ *Corresponding Author: jean-sebastien_deschenes@uqar.ca
}

\begin{abstract}
Laboratories are an important and integral part of the engineering curriculum and should be well integrated into a coherent learning path. In a distance learning context, the development and integration of remote laboratories represents an important technical challenge. Consequently, it is sometimes observed that the pedagogical and socio-affective aspects are neglected in the development of such laboratories. In this project, the objective has been to develop a flexible and reusable model (a full pedagogical package) designed to support the development of remote laboratory modules as well as providing an "ideal" distance learning environment that effectively supports teamwork between geographically dispersed students.

The proposed model is partly inspired from the problembased learning pedagogy with emphasis on collaboration, mutual training and peer support. Particular efforts were made to minimize the need for supervision. It is designed to make full use of the tools and flexibility provided by an existing learning management system (LMS) such as Moodle. The collaborative approach on which relies the completion of pre- and post-lab team documents is first discussed followed by a presentation of the other elements that are included in the model. The full concept is then summarized graphically, linking each module contents to their cognitive and socio-affective objectives. In addition to its usefulness as a guide to help developers design their remote laboratory interfaces, the model provides a convenient way to evaluate different aspects of a project (including teamwork and problem resolution processes) in addition to the actual learning objectives.
\end{abstract}

Keywords: Remote laboratories, Distance learning, Pedagogical package, Learning Management System, Peer collaboration and support.

\section{INTRODUCTION}

Latest trends in engineering education involve stronger emphasis on problem-solving skills and the development of the engineer himself rather than only on the development of knowledge [2]. Problem-based learning (PBL) can do well on this avenue, and its philosophy has been adapted for engineering courses on several occasions [2][18][12]. Such courses revolve around problems that are discussed (and solved) in groups, while the supervisor acts mainly as a facilitator. His guidance progressively decreases as the students increase their competence and autonomy in a process referred to as "scaffolding" [9]. The approach emphasizes problem-solving skills, peer collaboration and communication of results [10][12].

This general idea has also been adapted to laboratories on multiple occasions [2] [4] [5]. The concepts of pre-lab and post-lab are almost always present. The objectives of these activities may vary from making simple preparatory calculations and quickly write a simple report to the actual integration of various course learning objectives. Building student autonomy can be achieved progressively with each laboratory activity. It can thus prove worthwhile to deploy as much energy in the development of these surrounding activities as in the laboratories themselves to better achieve student learning and skill development objectives (learning path).

In a distance learning context, the integration of remote laboratories represents an even more important challenge [6]. In addition to the already very challenging technical aspects, the pedagogical and socio-affective elements pose significant difficulties and are sometimes neglected [15]. In this work, a particular emphasis was put on these aspects, in the development of a supportive pedagogical package to help design such remote experiments. Significant effort was put on reducing the supervisor interventions, thereby promoting self-reliance and peer support, while allowing an entirely flexible schedule for the realization of the laboratories by the students (even in the night, possibly, or week-ends).

CEEA13; Paper 048

Montreal, QC; June 17-20, 2013 


\section{THE ENVIRONMENT}

The first step in the development of the pedagogical package was to determine which format for the interface or virtual environment would be best suited to support our pedagogical objectives. Similar projects have made good use of open-source (cloud-based) sharing platforms and videoconference [17]. The ECHO project relied on the presence of the supervisor during most steps and the unregulated nature of the tools made tracking the students progress difficult and also prevented the introduction of scaffolds directly into the platform. Others have decided to build the interface, using development software like LabVIEW, adding to the technical challenge [3][15]. Both approaches have great potential but require either extensive supervision or developing skills and may be difficult to standardize and share. A third solution has been to integrate all the pre and post laboratory assignments into a learning management system (LMS) as in [4]. We believed this solution to be our best option for three reasons.

First, a LMS is directly attached to (and supported by) the teaching institution. It is already familiar to the students thereby reducing one element of perceived remoteness. Secondly, the chosen LMS is highly configurable. The content is arranged into blocks that can be easily modified, copied or adapted to the specifics of each laboratory. It is also very easy to include instructions and resources. And finally, all the necessary tracking functions are built into the LMS so that the supervisor can easily track each team's progress and provide timely feedback. Built-in functions also include communication tools as well as collaboration modules such as a wiki, glossary and database activities.

Specifically, Moodle is one of the LMS supported at our institution (UQAR) as well as many other institutions in the province, therefore inter-institution collaboration projects are easier to realize. Moodle also has a highly configurable interface, a wide range of tools and activities and allows the possibility of including external resources to the interface. It is also widely used throughout the world, making the proposed model accessible and easy to share.

\section{THE MODEL: COLLABORATIVE APPROACH}

The proposed model emphasizes collaboration over cooperation between team members. The distinction lies in the participation and role of each team member in the resolution of a common problem. Cooperation implies a "division of labor among participants", while collaboration adds another dimension to the interactions by involving a "mutual engagement of participants in a coordinated effort to solve the problem together" [14]. The mutual engagement between team members can be encouraged and nurtured to reach a point where the participants become a resource for each other, building knowledge through mutual training [11].

The pre-lab and post-lab activities can provide good opportunities to favor collaboration and the development of the learning processes at the participant's own pace. The following sub-sections present the pre-lab team document and the post-lab team report while the other supporting activities are described in section 4 .

\subsection{Pre-lab team document}

While pre-lab assignments are very common, the idea for the proposed pre-lab team document comes from another definition of collaboration as "a coordinated, synchronous activity that is the result of a continued attempt to construct and maintain a shared conception of a problem" [14]. The use of the words construct and maintain suggests a building, or foundations. The pre-lab document serves that purpose by proposing a concrete environment for the team to record observations, deposit documents and make revisions and, generally, share input. It also offers a more socio-affective way of maintaining a shared conception of the problem by creating a communal space for the teams to share, exchange and record information and opinions, receive feedback and also plan their interactions.

Providing a structured environment for the teams facilitates the introduction (and eventual removal) of scaffolds. At the start of a sequence of laboratories, the structure is very specific, with a lot of instructions and mandatory fields to be filled. It is designed to gradually evolve toward a less structured, more stimulating environment. This process of scaffolding and fading is believed to be necessary in a distance learning context where the supervisor (or tutor) is mostly absent.

Using the Database activity, a template is proposed to the students where fields have to be filled either individually or in collaboration. The document is divided into three sections, each with its own objectives.

In the first section, each participant is invited to fill the fields with personal information. The goal of this section is for the participants to get better acquainted, removing another layer of remoteness. In a classroom setting, the tutor would act as a facilitator, guiding the learning process [9]. Here, in a distance learning context, the role of facilitator is also provided by peers.

Individual accountability is a very important aspect that needs to be developed. Encouraging the participants to begin the reflection on their capacities and personal goals early in a project makes the final self-evaluation and peerevaluation easier. 
The second section consists of a collection of protocols and procedures that have been elaborated and approved as a team. This section and the following are built in a way that records not only the final version but also the process by which the team has reached an agreement. The final versions of the protocols serve as a contract between the participants ensuring that each member will be participating and productive. The supervisor will be able to monitor the teams collaborative process and offer guidance or feedback through a commentary function embedded in the activity.

The third section is the pre-lab section itself, and consists of fields where the teams record their efforts to be prepared for the laboratory. The pre-lab may include calculations, simulations, documentary research and/or plan the manipulations and is specific to each laboratory. This section is filled following a redaction and collaboration protocol elaborated in the second section. This protocol will include which communication tools will be used to complete the assignment. The pre-lab assignment is also tied to other activities, some of which are individual.

Finally, access to the laboratory module itself is conditional to the approval, by the supervisor, of the prelab document.

\subsection{Post-lab team report}

Post-lab assignments consist of two elements: the laboratory report, featuring the results, interpretation and discussion but also a sharing platform, where each team is encouraged to prepare and present its results to the community, inviting feedback and peer evaluation.

The post-lab report is conceived as a mirror activity relative to the pre-lab document. Developed using the database activity, it is a winding down of the project where lose strings are tied, results are discussed and each members participation, appreciation and contribution is evaluated in reference to the lab's objectives and the selfevaluation done during pre-lab.

\section{THE MODEL: OTHER ELEMENTS}

Individual lessons, the LMS and communication tools are not inherently collaborative tools. They support collaboration and as such are necessary but can be replaced by other activities, provided that the associated cognitive and socio-affective objectives are met. The following sub-sections describe briefly some of the most important supportive element.

\subsection{Individual Lessons}

The model includes some amount of individual work by the participants. Most of it is done during pre-lab and serves many purposes.

Lessons, in Moodle, are packages of content and questions arranged in a more or less flexible path. Using this particular activity for delivering content allows for better support and feedback. Stimulating individual reflections, research and learning helps to bring each participant to a level where their contribution to the team is meaningful.

There are three types of lessons planned in the model, each with its own objectives. The first lessons aims to introduce the environment and present each resource and activity in the proper context. The second lesson reviews the theoretical background necessary to complete the laboratory and finally, the third provides individual guidance for completing the pre-lab assignment.

\subsection{Communications}

As defined earlier, effective collaboration is done through synchronous communication intended to maintain a shared conception of a problem [14], thereby enhancing the feeling of "presence". However, "real-time" interactions can be unpractical with dispersed members. Therefore, it is optional for the pre- and post-lab assignments but required for the laboratory itself.

The completion of the pre-lab section includes obligatory discussions in the forums along with facultative chat sessions and videoconference. Again, using the communication tools included with Moodle allows the recording of discussions and facilitates feedback from peers and the supervisor.

On the whole, communications are planned to maximize the effectiveness of the whole model. The objectives to be attained are numerous, the feeling of "presence" being only one aspect of the experience. It is believed that using this argument for developing a fully synchronous model would have limited other aspects such as activities that require planning and reflection (i.e. the laboratory preparation and the lab report). Asynchronous communications namely, the forums and commentaries are thus preferred for long term discussions, particularly for the pre-lab assignment and contacts with the supervisor.

\subsection{Result sharing and peer evaluation}

Communication skills are also important to the development of an engineer. At the end of the laboratory the teams are encouraged to produce a recording or exposé of their results and share it with their peers. Each team's work is open for comments (moderated), feedback and discussion. The format, length and content is left voluntarily open to stimulate creativity and independent 
thinking and, hopefully, generate more feedback and inspire even better presentations.

This concludes the presentation of the most important features of the proposed model. The next section will present the main objectives to be attained in a graphical format.

\section{THE FULL MODEL: A GRAPHICAL REPRESENTATION OF THE OBJECTIVES TO BE ATTAINED}

Determining outcomes for any course or laboratory is necessary for a successful integration. Cognitive, socioaffective and motor objectives pursued by the laboratory may be varied and numerous and therefore must be clear and easy to evaluate.

In the proposed model, each activity has been associated to one or more objective which were derived (loosely) from Bloom's taxonomy for the cognitive and affective domains [1]. Another major inspiration has come from the Fundamentals Objectives of Engineering Instructional Laboratories [7], themselves derived from ABET criteria.

The six levels of cognitive skills are 1. Knowledge, 2. Comprehension, 3.Application, 4. Analysis, 5. Synthesis and 6 . Evaluation. Those levels are sufficiently clear by themselves and were affixed "as is" to each activities.

Socio-affective and psychomotor skills are a little bit more difficult to evaluate and quantify. Again using Bloom's Taxonomy as a reference we found the proposed levels to be too imprecise to use directly and so, for a more comprehensive list of objectives, we turned to the thirteen objectives that were established during a colloquy in San Diego in 2002 [7].

Of the thirteen objectives listed, six objectives have a "significant component of the affective domain, i.e., behavior and attitudes: learn from failure, creativity, safety, communications, teamwork and ethics in the laboratory." [8]. Lumping safety with learning from failure and ethics we obtain our first socio-affective category of objectives which we call "authenticity and accountability". We chose to combine creativity with communications which becomes "Communication" leaving "Team work" as the last category.

Since it may be difficult to classify socio-affective abilities and behavior according to any order of importance without further research beyond the scope of our current project, we elected to consider each of the above mentioned categories as an independent set of objectives while keeping in mind that they are interconnected.

From these objectives, that deal with the cognitive domain, of course, but also the affective domain and psychomotor aspects, we defined a graphical set of goals for each major activity, which we present here with commentaries.

Figures 1 and 2 present a summary of the pre-lab activities and the levels of cognitive skills (Fig. 1) and the socio-affective aspects (Fig. 2) that are targeted.

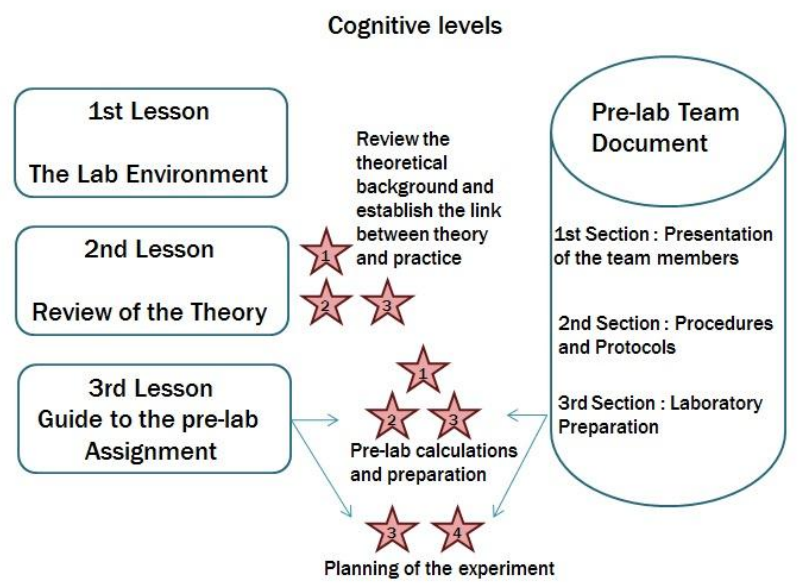

Fig. 1. Cognitive skills stimulated in the pre-lab section

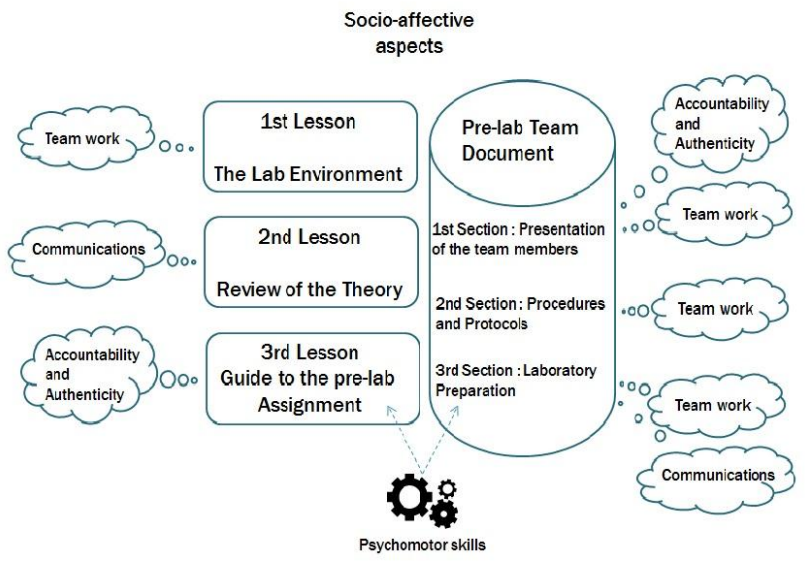

Fig. 2. Socio-affective aspects stimulated in the pre-lab section

As we can see from Fig. 2, all three categories of objectives from the socio-affective domain are covered in the pre-lab section. The main focus of this section, however is the building of the team while, at the same time, bringing the cognitive skills to the highest level possible.

Psychomotor skills are included in Fig. 2 and linked to the activities, with dotted arrows, to indicate that those skills are indeed stimulated in the pre-lab activities but only marginally and are not discussed further in the present article. 
Post-lab activities, particularly the laboratory report, aims to complete the cognitive process: All six skills of the cognitive domain are stimulated (Fig. 3).

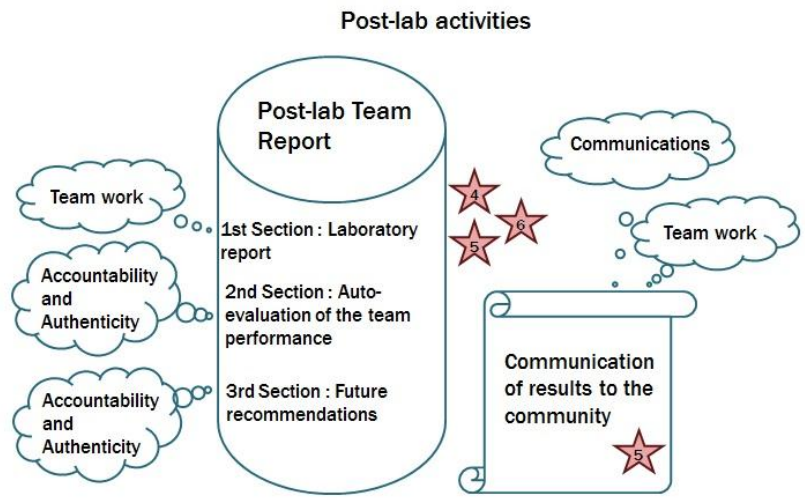

Fig. 3. Post-lab activities and associated objectives

Finally, other post-lab activities include self-evaluation, peer evaluation and the communication of results. Those activities stimulate introspection, objectivity and creativity. This section of the model is arguably one the most important for the development of the engineer as it aims to foster real life skills and abilities bridging the gap between theory and practice.

\section{CONCLUSION}

This paper presented a reusable model for developing remote laboratories, providing convenient means for the evaluation of transversal aspects of a project in addition to the actual learning objectives. The model emphasizes on collaboration, mutual training and peer support, and aims at minimizing the supervisor interventions, as the remote activities are to possibly take place arbitrarily during the week (even at nights). It is adaptable to various situations and built to better support the learning process. Future work will imply testing the concept with students and see the impacts on motivation and learning effectiveness.

\section{Acknowledgements}

The authors would like to thank the Université du Québec and the FODAR, the École de Technologie Supérieure de Montréal, the Télé-Université du Québec, the Université du Québec à Rimouski and the Université du Québec en Outaouais for funding this research.

\section{References}

1. Anderson L. and Krathwohl D.A., 2001. Taxonomy for Learning, Teaching and Assessing: A Revision of Bloom's
Taxonomy of Educational Objectives. New York, NY: Addison Wesley Longman.

2. Cheville A. and Bunting C. 2011. Engineering Students for the 21st Century : Student Development through the Curriculum. Advances in Engineering Education, 2011, pp. 1-37.

3. Dodo Amadou M. M., Saliah-Hassane H. and Saad M. Laboratories in Engineering Courses via Internet. International Conference on Engineering Education and Research "Progress Through Partnership", Olomouc and Bouzov Castle, Czech Republic, June 27-30, 2004, pp. 1089-1095.

4. Donzellini G.. and Ponta D. 2007. The Electronic Laboratory: Traditional, Simulated or Remote. Advances on Remote Laboratories and E-learning Experiences. Deustso Publicaciones, pp. 35-62.

5. Dormido R., Vargas H., Duro N., Sanchez J., DormidoCanto S., Farias G., Esquembre F. and Dormido S. 2008. Development of a Web-Based Control Laboratory for Automation Technicians: The Three-Tank System. IEEE Transaction on Education, vol. 51, no. 1, pp. 35-44.

6. Fabregas E., Farias G., Dormido-Canto S., Dormido S., Esquembre F. 2011. Developing a Remote Laboratory for Engineering Education. Computers and Education, vol.57, pp. 1686-1697.

7. Feisel L. and Peterson, G.D. A Colloquy on Learning Objectives for Engineering Educational Laboratories. 2002 ASEE Annual Conference and Exposition, Montreal, June 16-19, 2002.

8. Feisel L. D. and Rosa A. J. 2005. The Role of the Laboratory in Undergraduate Engineering Education. Journal of Engineering Education, January 2005, pp. 121-130.

9. Greening T., 1998. Scaffolding for Success in ProblemBased Learning. Medical Education Online, vol. 3, pp. 115.

10.Kwan C.Y., 2000. What is Problem-Based Learning (PBL)? CDTL Brief, vol. 3, no. 3, pp. 1-2.

11.Linard M. Quelles nouvelles compétences des acteurs de la formation dans le contexte des TIC? Éducation permanente, 12, 19-47

12.Litzinger T., Lattuca L., Hadgraft R., Newstetter W. 2011. Engineering Education and the Development of Expertise. Journal of Engineering Education, January 2011, pp. 123150 .

13. Müller D. and Erbe H.-H. 2007. Collaborative Remote Laboratories in Engineering Education: Challenges and Vision. Advances on Remote Laboratories and E-learning Experiences. Deustso Publicaciones, pp. 35-62.

14. Roschelle J. and Teasley S. D. 1995. The Construction of Shared Knowledge in Collaborative Problem-solving. O’Mailley (Ed.): Computer Supported Collaborative Learning, Springer Verlag, Heidelberg, pp. 69-97.

15. Saliah-Hassane H., Laboratoires en ligne. Accessed May 2012 from http://www.profetic.org/dossiers/

16. Saliah-Hassane H., Dumont-Burnett P. and Loizeau C. Design of a Web-Based Virtual Laboratory Instrument Measurement Interface. International Conference on 
Engineering Education, Oslo, Norway, August 6-10, 2001, pp. (8D1-12)-(8D1-16).

17. Villardier L., Saliah-Hassane H. and Probst W. ÉCHO: A Configurable Remote Training and Monitoring Real-Time Environment. International Conference on Engineering Education. Valencia, Spain, July 21-25, 2003, 8 pp.

18. Woods et al. Developing Problem Solving Skills: The McMaster Problem Solving Program. Journal of Engineering Education Volume 86, Issue 2, 1997,pages 75-91. 